ज्ञ FRANÇAISE

$\supset$ DE

딜 PEAGOGIE

\section{Revue française de pédagogie}

Recherches en éducation

156 | juillet-septembre 2006

Les espaces locaux d'interdépendance entre

établissements : une comparaison européenne

\title{
Positions, logiques d'action et inégalités entre les écoles d'un arrondissement de Budapest
}

Relations of interdependency between schools in a Budapest district: positions, strategies and differences

Relaciones de interdependencia entre las escuelas de un distrito de Budapest: posiciones, lógicas de acción y desigualdades Gegenseitige Unabhängigkeit zwischen den Schulen eines Budapester Bezirks: Stellungen, Handlungsprinzipien und Ungleichheiten

Iván Bajomi, Eszter Berényi, Gábor Eröss et Anna Imre

\section{OpenEdition}

\section{Journals}

Édition électronique

URL : http://journals.openedition.org/rfp/328

DOI : $10.4000 /$ rfp.328

ISSN : 2105-2913

Éditeur

ENS Éditions

Édition imprimée

Date de publication : 1 juillet 2006

Pagination : $39-50$

ISBN : 978-2-7342-1060-3

ISSN : 0556-7807

\section{Référence électronique}

Iván Bajomi, Eszter Berényi, Gábor Eröss et Anna Imre, « Positions, logiques d'action et inégalités entre les écoles d'un arrondissement de Budapest », Revue française de pédagogie [En ligne], 156 | juilletseptembre 2006, mis en ligne le 27 septembre 2010, consulté le 30 avril 2019. URL : http:// journals.openedition.org/rfp/328; DOI : 10.4000/rfp.328 


\title{
Positions, logiques d'action et inégalités entre les écoles d'un arrondissement de Budapest
}

\author{
Iván Bajomi, Eszter Berényi, \\ Gábor Eröss, Anna Imre
}

Les logiques d'action des établissements hongrois sont très diversifiées et se déploient notamment lorsque les parents sont amenés à faire - en principe librement - un choix d'école pour leur enfant de six ans, choix déterminant du point de vue de leur future carrière scolaire. Aussi, la concurrence entre établissements est souvent exacerbée et les positions des écoles sont inégalitaires. Ces inégalités ne découlent pas seulement de la composition sociale du secteur ou de la composition sociale réelle des effectifs d'élèves, mais aussi de la position géographique de l'école à l'intérieur de l'espace local, de sa taille, ou encore des liens plus ou moins privilégiés qu'entretiennent les chefs d'établissement avec la mairie. Certaines écoles essayent d'attirer surtout les enfants issus des couches sociales supérieures, d'autres sont contraintes de se spécialiser dans l'accueil des enfants en difficulté et il existe également des écoles bi-polaires qui, tout en ouvrant des classes d'élite, ont également des classes dites "normales " scolarisant un nombre important d'élèves défavorisés. Outre les établissements caractérisés par les stratégies ci-dessus mentionnées, il existe aussi quelques écoles non mobilisées.

Descripteurs (TEE) : gestion d'établissement scolaire, inégalité sociale, Hongrie, répartition géographique, ségrégation, tsigane

Q uatre particularités caractérisent la situation hongroise : la sélection-différenciation très précoce des élèves, la grande proximité entre les principaux acteurs locaux de régulation scolaire et les établissements, la baisse vertigineuse du nombre d'élèves et, enfin, la ségrégation, qui frappe certaines catégories d'enfants, notamment ceux d'origine tsigane, renforcée par les effets pervers de l'action « bienveillante » de certains services psychopédagogiques.

\section{INTRODUCTION}

Depuis une quinzaine d'années, le système éducatif hongrois fonctionne de façon décentralisée (1). Dans la capitale, la gestion des établissements scolaires du premier degré et d'une partie des écoles secondaires est ainsi assurée par les municipalités d'arrondissement. S'il existe des écoles privées 
depuis le changement de régime, à peu près $90 \%$ des écoles fondamentales relèvent des municipalités. Celles-ci reçoivent du gouvernement un financement en fonction du nombre d'élèves, mais elles peuvent, lorsqu'elles en ont la volonté et les moyens, y ajouter un financement additionnel. Certaines écoles peuvent ainsi percevoir jusqu'à 30 , voire $40 \%$, de fonds supplémentaires. À ces inégalités entre écoles de municipalités différentes s'ajoutent des inégalités au sein même des municipalités.

Dans les villes, ces inégalités résultent aussi bien de l'implantation géographique des écoles que des logiques d'action mises en œuvre par les établissements en réaction à leur position « de départ », plus ou moins favorable, c'est-à-dire en fonction de la composition socio-économique de leurs secteurs de recrutement respectifs. Mais elles doivent aussi tenir compte des orientations de la municipalité et des caractéristiques de la population d'élèves de l'arrondissement tout entier, voire des arrondissements voisins (dans le cas d'écoles situées en périphérie d'arrondissement). L'attention portée aux parents est due notamment au libre choix de l'école qui caractérise désormais le pays. Aussi le choix fait par les parents, lorsque leur enfant entre à l'école à l'âge de six-sept ans, est-il décisif. Le cas hongrois témoigne du fait qu'une politique d'éducation libérale (2), conjuguée à une chute démographique dramatique, peut mener à des relations extrêmement concurrentielles provoquant des inégalités profondes, même en l'absence d'établissements privés.

Dans cet article visant à présenter certains résultats d'une enquête empirique (3) effectuée conformément aux orientations générales du projet de recherche européen Reguleducnetwork nous décrirons brièvement les caractéristiques socio-démographiques de l'arrondissement étudié, avant de mettre en évidence les relations d'interdépendance entre les écoles de même que les inégalités entre établissements scolaires et entre élèves. Sur la base des informations recueillies, nous montrerons à quel point les stratégies élaborées par les écoles peuvent être différentes, tandis que subsistent encore quelques établissements scolaires non-mobilisés qui, malgré la forte baisse démographique, conservent un mode de fonctionnement traditionnel. Le concept de logique d'action nous permettra d'analyser la situation en termes de causalité circulaire et de reproduction des inégalités sans négliger l'ensemble des facteurs, nombreux, qui peuvent biaiser, aggraver ou contrebalancer les inégalités et les stratégies de départ dans le contexte de « libre » marché scolaire (4).

\section{Les caractéristiques sociales du territoire étudié}

L'arrondissement étudié est un quartier ouvrier traditionnel de Budapest (5). Une partie considérable de sa population s'est appauvrie au cours des quinze dernières années et le taux de chômage est assez élevé, mais néanmoins inférieur à celui des banlieues des métropoles occidentales ou de l'Est de la Hongrie. En 2002, «Paladomb» était peuplé d'environ 78000 habitants et avait perdu en quinze ans $22 \%$ de sa population. Le dépeuplement a frappé particulièrement la classe d'âge étudiée des six-quinze ans. La population est actuellement plus jeune que la moyenne de Budapest ou que la moyenne nationale, mais la tendance, comme ailleurs, est au vieillissement, ce qui influence fortement les relations interétablissements.

Les classes populaires sont nombreuses dans cet arrondissement, mais un niveau important de mixité urbaine subsiste tout de même. Une proportion élevée, soit $70 \%$ de la population, habite dans des cités souvent délabrées (mais pas trop insécurisées). Tout comme dans le reste du pays, les Tsiganes de Paladomb sont considérés comme une population «à problèmes " bien qu'ils soient sédentarisés depuis des générations et très majoritairement hungarophones. Selon les derniers chiffres officiels, datant de l'année scolaire 1992-1993, 6,5\% des élèves étaient répertoriés comme étant d'origine tsigane. Depuis cette date, leur proportion a du augmenter. Ces enfants se répartissent d'une façon très inégale entre les différentes écoles à cause de la ségrégation relativement importante de l'habitat, mais surtout du mode de fonctionnement du marché scolaire.

\section{L'offre scolaire dans l'arrondissement}

En Hongrie, les enfants entrent à l'école fondamentale à l'âge de six ou sept ans. Ces écoles couvrent huit années d'étude mais, depuis la chute du communisme, il est possible de les quitter après les quatre ou six premières années pour s'inscrire dans de lycées proposant huit ou six ans d'études. De tels lycées n'existent cependant pas dans l'arrondissement étudié. En 2001-2002 et 2002-2003, il existait dix-sept écoles fondamentales gérées par la municipalité locale et une seule école privée (pour des élèves ayant un handicap mental léger).

Du point de vue de leur localisation géographique, les écoles fondamentales présentent des situations très variées. Elles peuvent être réparties en trois catégories. Les six écoles du centre de Paladomb sont 


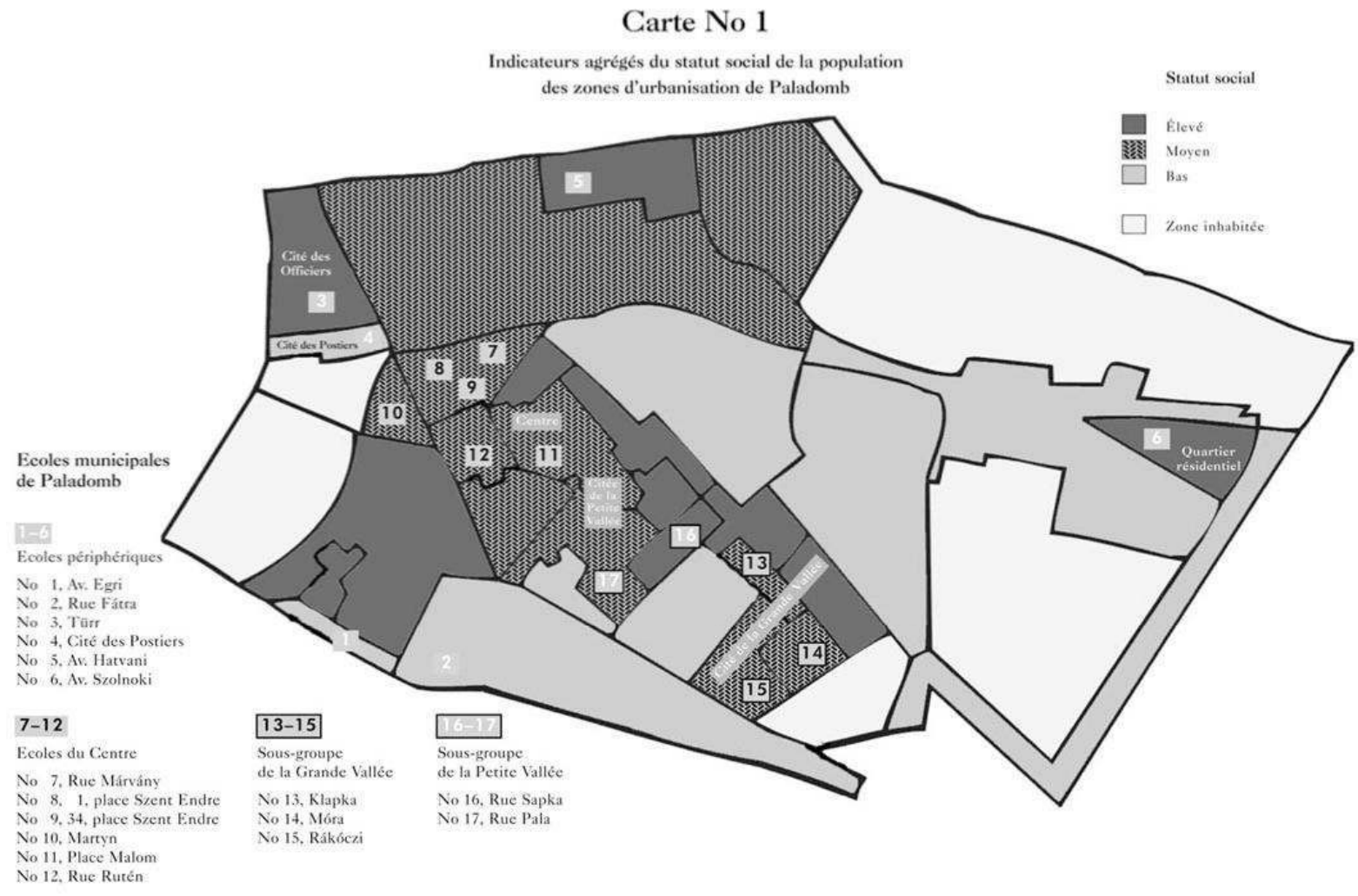

faciles d'accès, non seulement pour les habitants de l'arrondissement mais également pour ceux des arrondissements voisins. Deux d'entre elles sont les écoles ayant traditionnellement la meilleure réputation. En revanche, cinq écoles peuvent être considérées comme périphériques. Situées à proximité des frontières de Paladomb, elles accueillent souvent beaucoup d'enfants venus des arrondissements limitrophes. Parmi elles, l'école de la Cité des Postiers est dans une situation particulière caractérisée par une importante ségrégation: située à la périphérie de l'arrondissement, elle dessert une ancienne cité d'employés de la Poste, très détériorée; ses élèves sont tous défavorisés et en très grande majorité d'origine tsigane. Tout près de cette école se trouve l'école Türr, qui dessert la cité des officiers, considérée comme un quartier d'élite (par rapport au reste de l'arrondissement) où habitent les enfants et les petits-enfants des officiers, dont bon nombre a fait des études supérieures. La dernière catégorie d'écoles dessert les deux grandes cités de l'arrondissement. Ces établissements scolaires forment deux sous-groupes : dans la très grande cité de la Grande
Vallée, se trouvent trois écoles presque voisines (6) ; deux autres établissements, situés à mi-chemin entre la Grande Vallée et le centre, desservent la cité de la Petite Vallée.

\section{Relations d'interdépendance entre les écoles observées}

Les relations d'interdépendances entre les écoles de l'arrondissement proviennent essentiellement de deux facteurs. D'une part, comme la municipalité gère et finance toutes les écoles fondamentales, à l'exception d'une seule d'entre elles (7), son action (parfois son inaction) et ses choix de politique éducative sont déterminants (8). Mais les interdépendances compétitives entre les écoles résultent avant tout des choix parentaux. En effet, chaque école de l'arrondissement dispose d'un secteur de recrutement ; les familles qui y habitent peuvent inscrire leurs enfants dans l'établissement de leur secteur; mais le fait qu'elles n'y soient pas obligées génère une concurrence entre écoles pour attirer les élèves. Ce phénomène est 
renforcé par la baisse du nombre des inscrits ces dernières années: " II n'y a pas d'intérêt commun. Mon intérêt est de survivre. L'intérêt de l'autre est la fermeture de mon établissement, ce qui lui permettra de survivre » (directrice, Centre).

Ce n'est que récemment que les membres du service scolaire essaient de contrebalancer les relations concurrentielles des établissements par un interventionnisme plus poussé. En effet, après le changement de régime au début des années 1990, les gestionnaires locaux du réseau d'éducation étaient en faveur de la concurrence et encourageaient la " diversité " du système, ce qui a favorisé la multiplication de "classes de niveau » et la sélection des élèves. À l'époque, pour ces fonctionnaires, les effets bénéfiques d'un marché scolaire allaient de soi. Les objectifs de la municipalité ont changé ces dernières années, mais ce soutien à la compétition a influencé le système d'interdépendance à long terme.

Au sein de l'arrondissement, les liens d'interdépendance se tissent notamment en fonction de la segmentation géographique et sociale du territoire. C'est parmi les écoles du centre, très proches les unes des autres, que l'on constate les relations les plus intenses ; ces établissements scolaires forment une première grappe d'interdépendance très concurrentielle. $\mathrm{Au}$ sein de la grappe des grandes cités, et de ses deux sous-ensembles, la concurrence est moins forte. Enfin, un troisième sous-ensemble regroupe le tiers restant des écoles, que l'on peut qualifier de "périphériques ". II est formé d'écoles isolées ou insérées dans des grappes différentes centrées sur des arrondissements voisins. De ce fait, ces écoles sont moins liées par des interdépendances compétitives avec les écoles des deux autres ensembles.

Compte tenu de la composition sociale des quartiers d'implantation des différents établissements (9), certaines écoles sont dans une position particulièrement favorable dans la lutte pour attirer les enfants issus de milieux favorisés (coir carte $n^{\circ} 1$ ). À l'inverse, les établissements tel celui de la Cité des Postiers partent avec de lourds handicaps. Cependant, presque la moitié des écoles sont en position socio-démographique moyenne, soit parce qu'elles sont situées en "zone moyenne " soit parce que leur secteur regroupe des zones d'urbanisation diverses. Leur situation de départ est donc similaire, ou moins extrême que dans un espace plus ségrégué (tels qu'il en existe dans certaines grandes agglomérations de l'Ouest de l'Europe). La concurrence directe pour l'attraction des élèves joue alors un rôle décisif, mais la lutte pour les ressources en provenance de la municipalité est elle aussi parfois féroce.

Puisque la baisse démographique est l'un des problèmes majeurs du secteur de l'éducation dans la Hongrie contemporaine, les variations des effectifs d'élèves constituent un indicateur particulièrement important de l'attractivité relative des établissements et de leur position dans les relations de concurrence. Bien que les effectifs d'élèves aient baissé significativement au cours des dernières années au niveau de l'arrondissement, quelques écoles centrales ont pu faire croître leurs effectifs, tandis que les écoles de la périphérie ont perdu le plus d'enfants.

Pour se faire une idée plus précise des rapports de force entre les écoles, il faut étudier l'origine géographique des élèves par rapport à la localisation de leur établissement, et en particulier la proportion des enfants originaires de l'arrondissement mais ne venant pas du secteur de recrutement officiel de l'école (Graphique 1).

Nos données montrent que ce sont les écoles des cités et une partie des établissements scolaires périphériques qui sont fréquentées avant tout par des enfants du secteur, tandis que c'est dans le centre que le taux d'élèves venant d'autres secteurs de l'arrondissement est le plus élevé. Notons aussi que les écoles du centre se caractérisent par une importante concentration d'enseignants dotés de diplômes universitaires et concentrent la plupart des filières et écoles d'élite : le centre de l'arrondissement exerce une attraction "d'excellence » sur les habitants des autres quartiers, et notamment sur ceux des deux grandes cités.

Les différences observées peuvent être imputées à des facteurs très différents, d'ordre géographique ou stratégique. On peut par exemple comparer les cas des écoles Márvány (centre) et Hatvani (périphérie). La première est une école très attractive; le taux d'élèves issus du secteur de l'école est particulièrement bas, mais comme le nombre d'élèves venus d'autres arrondissements l'est également, l'établissement ne pose pas de problème de financement à la municipalité (10). À l'inverse, Hatvani, en raison de sa situation géographique décentrée, est l'une des écoles qui desservent le plus leur propre secteur. En effet, la grande majorité des enfants du secteur est bel et bien scolarisée dans cette école (et représente environ $55 \%$ du total de sa population d'élèves). Mais, comme il n'y a plus assez d'enfants en âge d'être scolarisés sur place et qu'il y a une grande cité de l'autre côté de l'avenue, c'est-à-dire dans l'arron- 


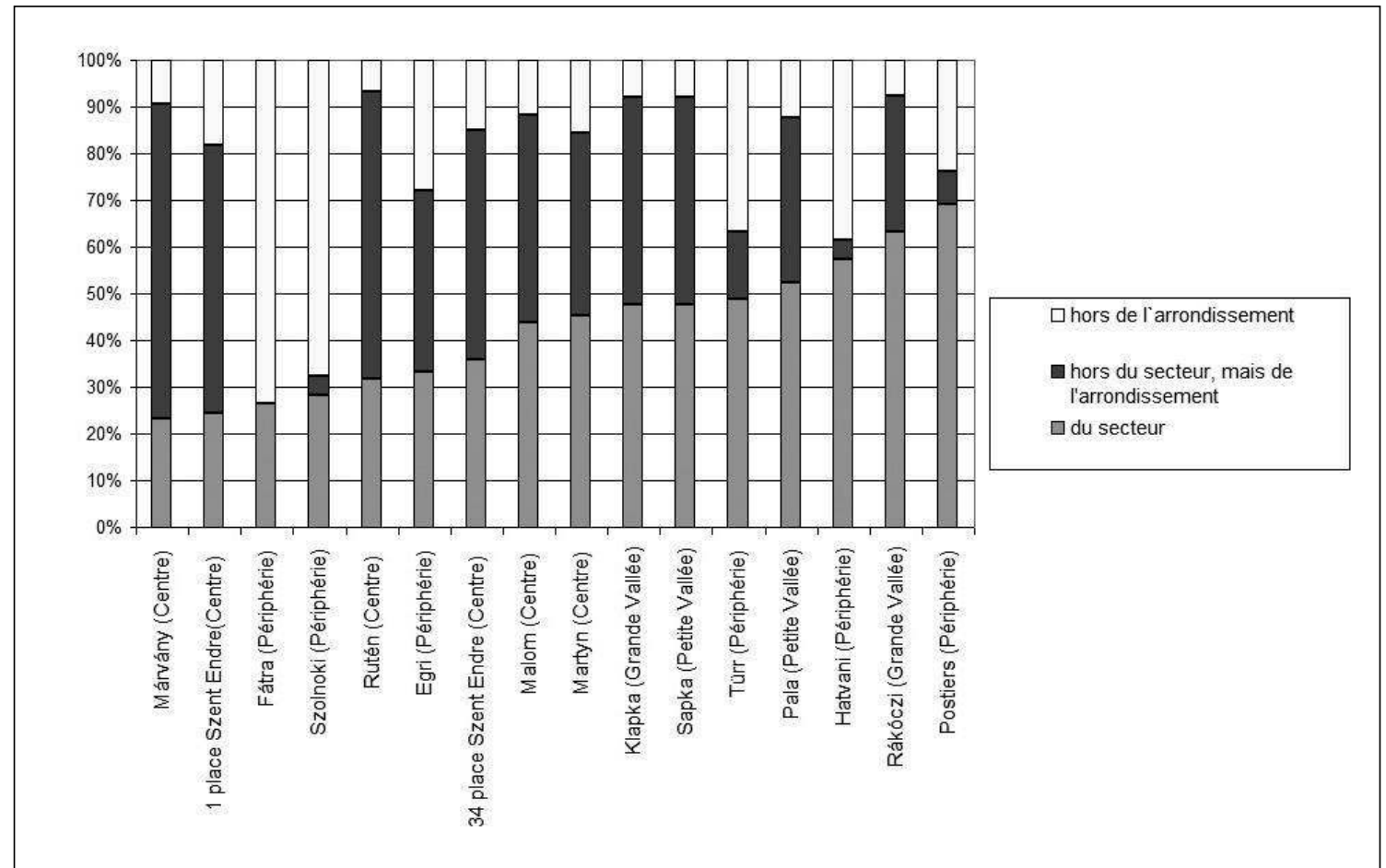

*L'école Móra n’a pas communiqué ses données et celles de Fátra sont partielles

dissement d'en face, les élèves qui ne sont pas issus du secteur de recrutement de l'école viennent essentiellement de l'arrondissement voisin et représentent $39 \%$ de sa population d'élèves. Or, la proportion élevée d'élèves issus d'arrondissements voisins met l'école en position de vulnérabilité (à moins que son directeur soit un élu municipal, comme c'est parfois le cas) car la municipalité est de moins en moins prête à financer la scolarisation d'élèves "étrangers " au territoire de Paladomb. Une localisation périphérique diminue donc la marge de manœuvre des écoles, d'autant qu'il est difficile à celles-ci d'attirer les enfants d'autres secteurs de l'arrondissement.

\section{Les logiques d'action des établissements}

En raison du libre choix de l'établissement scolaire, du système étatique de financement per capita, des faveurs qu'accorde la municipalité à certaines écoles plutôt qu'à d'autres et de la chute rapide du nombre d'élèves, la concurrence entre les écoles est exacerbée. Les positions de départ des écoles (définies par la composition sociale des habitants de leur secteur) déterminent en grande partie la stratégie "d'attraction " d'élèves qu'elles sont en mesure de mettre en œuvre.

La logique d'action des écoles en matière d'inscription répondait jusqu'à une date toute récente à deux objectifs parfois contradictoires : d'une part, il fallait attirer un maximum d'élèves (compétition de premier ordre), et, d'autre part, il s'agissait d'attirer - si possible - des élèves peu problématiques et issus de milieu sociaux non défavorisés (compétition de second ordre). Le volume et la qualité des élèves accueillis lors des inscriptions effectuées à l'âge de six ans représentent dès lors des enjeux clés. La compétition sur ces deux plans se développe dans un cadre légal interdisant l'organisation de concours d'admission. Cependant, certains établissements dotés de filières sélectives (classes de niveau, classes «sport-étude ", etc.) n'hésitent pas à organiser une épreuve d'admission qui ne dit pas son nom. La 
municipalité feint d'ignorer ces pratiques connues de tous. En effet, les écoles qui violent la règle de la manière la plus ostentatoire sont aussi celles qui scolarisent les élèves de l'élite locale ou dont les directeurs ont leur entrée ou même leur siège au sein du Conseil municipal et de la Commission scolaire.

À la sélection par épreuve d'admission correspond une offre scolaire diversifiée. À Paladomb, coexistent huit types différents de classes de niveau, filières plus ou moins élitistes et compétitives dont certaines, comme "la classe préparatoire pour le lycée ", apparaissent dans plusieurs écoles (11) en réponse aux demandes des parents d'enfants de six ans, déjà soucieux de l'offre du cycle supérieur (dix-quatorze ans) que pourra leur proposer l'établissement. Selon certains chefs d'établissement, les filières spéciales sont devenues une valeur en soi aux yeux de beaucoup de parents : d'après ces directeurs, une conviction collective s'est développée chez les parents selon laquelle c'est en inscrivant leurs enfants dans une classe spécialisée quelconque qu'ils peuvent contribuer le plus à leur réussite future, protégés qu'ils seront de la présence dérangeante de camarades « difficilement contrôlables ».

À l'autre bout de la hiérarchie sociale et scolaire, trois types de classes sont destinées aux enfants «à problèmes" (classes d'orthophonie, classes "intégrées " et classes de « remise à niveau » à effectif restreint). L'ouverture de celles-ci peut devenir une ressource importante dans la compétition inter-scolaire, puisque le financement forfaitaire national pour ces classes représente le double du financement des élèves inscrits en classes "normales". Ainsi, ces enfants défavorisés constituent de plus en plus une ressource convoitée : ils comptent double.

Toutefois, la réglementation nationale limite ces diverses stratégies en direction des familles aisées ou des enfants « à problèmes » en prescrivant le maintien d'au moins une classe normale, dans laquelle l'école doit accueillir les enfants de son secteur. Cette réglementation favorise la bipolarité de certains établissements attractifs qui proposent des classes « normales " cohabitant avec des filières d'élite. L'obligation de garder au moins une classe "normale » apparaît alors comme une entrave à la stratégie d'attraction de l'école (ou au mieux, un moyen de maintenir des effectifs d'élèves et d'enseignants suffisamment importants pour ne pas être menacé de fermeture). Ces classes peuvent dès lors fonctionner comme des voies de garage. Les élèves d'origine défavorisée y ont en général des enseignants moins qualifiés, tandis que les prestations assurées ne tiennent guère compte des besoins spécifiques des enfants. La bipolarité peut exister aussi dans les écoles ayant des classes "normales " à côté de classes de rattrapage à effectifs restreints ou d'orthophonie. Les classes de ces écoles - qu'il s'agisse de la première ou de la deuxième configuration - sont constituées selon un schéma similaire à celui des «bonnes " et des "mauvaises " classes des collèges français de banlieue (van Zanten, 2001, p. 120-125). Les «bonnes classes » ou classes «protégées " permettent à l'école de ne pas perdre des effectifs tout en rendant possible aux parents des classes moyennes (cadres, employés, fonctionnaires, professions intellectuelles petits entrepreneurs, etc.) de "coloniser " l'établissement de leur quartier et aux enseignants de venir "s'y ressourcer", tandis que les "mauvaises classes " contribuent au maintien des effectifs. Dans les écoles bipolaires, le statut social du chef de famille varie énormément d'une classe à l'autre.

En définitive, on peut distinguer trois logiques d'action qui tiennent compte de façon variable des demandes des familles de classe moyenne, de la régulation exercée par la municipalité, et des logiques d'action des autres établissements, les deux premiers facteurs pouvant entrer en tension.

a) Certaines écoles tiennent compte avant tout des priorités de la municipalité, en offrant des classes de rattrapage ou des classes d'orthophonie. Elles n'ont d'autres ressources à mobiliser que le financement forfaitaire par élève (provenant de l'État, mais versé par la mairie). Les cinq écoles de ce type ont des secteurs plutôt défavorisés ou moyens.

b) Certaines écoles tiennent compte des attentes des parents d'élèves des classes moyennes de leur environnement autant que des priorités de la municipalité. Ce sont surtout les écoles bipolaires les plus grandes qui, tout en cherchant à attirer surtout des élèves de statut social élevé, se montrent très sensibles aux volontés municipales comme celle de réduire le nombre d'élèves hors arrondissement : leur logique d'action implique en effet un surcoût de financement occasionné par les classes "d'élite". Dans les cinq établissements de ce type, on rencontre souvent des classes dites normales qui sont en réalité des classes abandonnées.

c) Certaines écoles ne font que le strict minimum en vue de respecter les priorités de la municipalité et se conforment aux attentes de l'élite locale. Les directeurs des deux écoles de ce groupe ont tendance à se représenter le système scolaire comme un marché. Ces acteurs pensent souvent l'action de 
la mairie en termes de contraintes inutiles, les empêchant de recruter encore plus librement les élèves qui conviendraient le mieux à leur logique d'action.

Il existe un lien entre ces trois types de logiques d'action et les types de localisation au sens où, pour appliquer une stratégie entièrement tournée vers les élites locales, il faut être situé dans le centre de l'arrondissement ou avoir un secteur de scolarisation plutôt favorable, que ce soit dans le centre ou en périphérie. De façon complémentaire, ceux qui doivent se contenter de suivre la municipalité se comptent parmi les perdants de la compétition entre écoles du centre ou parmi les établissements ayant les secteurs les moins favorables. Entre ces deux extrêmes, les grandes écoles de la grande cité sont typiquement (mais pas exclusivement) celles qui peuvent et veulent appliquer une stratégie intermédiaire (de type « $b »$ ).

De même, des liens forts existent entre les logiques d'action et les stratégies de direction visant à une meilleure "gestion " de l'hétérogénéité des élèves. Cela est très clair dans le cas des écoles bipolaires. Au total, trois pratiques de gestion de l'hétérogénéité émergent: la logique de spécialisation, la logique de bipolarisation et la logique de traitement ordinaire.

Les directeurs «actifs» qui suivent la logique de spécialisation essayent - en fonction de l'emplacement de leur établissement - soit de faire de leur école un établissement spécialisé dans l'éducation des élèves en difficulté qui, dans la plupart des cas, sont des enfants défavorisés («spécialisation-soutien": classes à effectif réduit, emploi d'un orthophoniste, de plusieurs psychologues scolaires, etc.), soit, au contraire, de répondre aux exigences des parents des couches sociales supérieures tout en rejetant les enfants «à problèmes » ("spécialisation-rejet » : examens d'entrée illicites mais tolérés par la mairie).

La logique de bipolarisation, qui suppose un mode de direction un peu moins actif, consiste à mettre en place une stratégie double : ouverture vers l'élite et vers les enfants en difficulté, en les maintenant de fait dans des classes séparées. Une partie des établissements abandonne les enfants défavorisés à leur sort dans les classes «normales » (bipolarité cachée), tandis que d'autres, tout en «institutionnalisant » la ségrégation, élaborent des stratégies actives de soutien qui leur servent de "ressources" dans la lutte pour les subventions (bipolarité ouvertement assumée).

Enfin, la logique de traitement ordinaire correspond à une attitude plutôt passive de la part de la direc- tion, celle-ci se contentant principalement des classes "normales". Seules les écoles ayant un secteur de scolarisation peu concurrentiel, de composition moyenne et peu menacé par la chute démographique peuvent se permettre de ne pas être actives. Autant dire que, d'année en année, le nombre d'écoles dont la direction pourrait être classée dans cette catégorie, diminue.

\section{LOGIQUES D'ACTION ET HIÉRARCHIE DES ÉTABLISSEMENTS}

II nous faut à présent examiner les liens complexes entre les logiques d'action des établissements et leur position dans l'espace local des inégalités de position (12). Nous avons vu qu'à cause du libre choix de l'école par les parents d'élèves, la composition sociale des écoles n'était pas identique à celle de leur secteur. Avant de mettre en évidence les logiques de différenciation entre écoles et la part des différents facteurs (emplacement, taille de l'établissement, stratégie de la direction, intervention de la municipalité, démographie, etc.), nous allons montrer l'étendue des inégalités entre écoles en termes de statut social des élèves scolarisés. Notre analyse concernant la composition sociale des élèves se base sur le niveau d'instruction des pères (13).

Dans le cas des quatre premières écoles, la moyenne du niveau d'instruction des pères est supérieure au baccalauréat. À l'autre "extrémité » de la hiérarchie, on retrouve les deux écoles «tsiganes", Rutén et la Cité des Postiers. Le niveau d'instruction des pères est assez élevé dans les écoles Fátra et Márvány : bien que situées pourtant dans des zones d'urbanisation dont la composition sociale est légèrement inférieure à la moyenne, elles drainent un nombre important d'enfants domiciliés à l'extérieur de l'arrondissement. Dans le cas de l'école Martyn, le statut social des parents est également significativement supérieur à celui que l'on s'attendrait compte tenu de la composition sociale de la population du secteur. La grande perdante de la compétition inter-scolaire est l'école Móra, qui bénéficie d'un secteur relativement " bon ", tandis que le niveau d'instruction des pères des élèves fréquentant cette école est inférieur à la moyenne de l'arrondissement. Nous pouvons suspecter ici l'effet de l'attrait des écoles du centre et de leurs « filières "; Móra, suffisamment grande pour ne pas être menacée de fermeture, ne souhaite pas leur emboîter le pas (14). 
Graphique 2. - Le niveau d'instruction moyen des pères des élèves par école

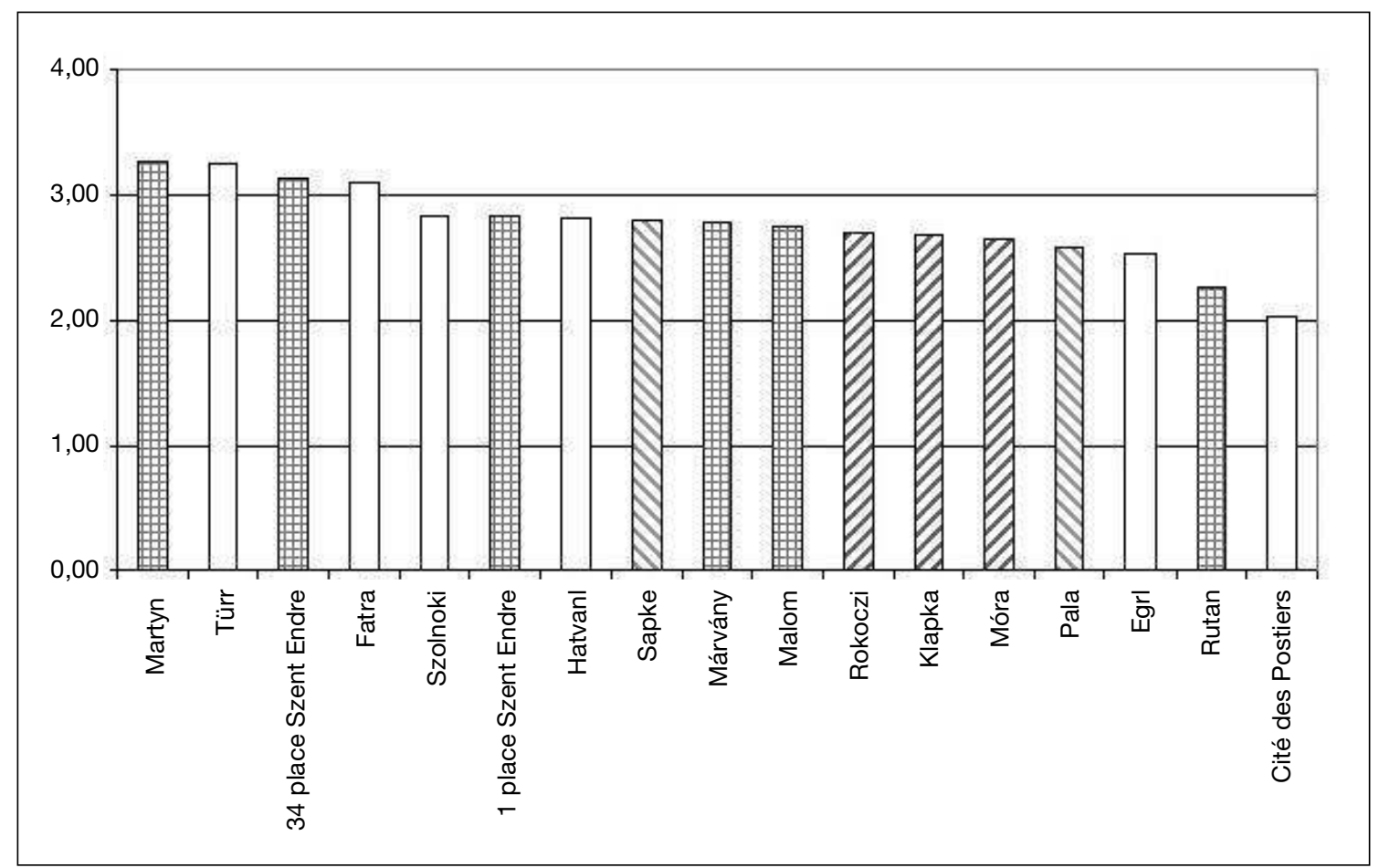

田 écoles du centre $-\nabla$ écoles de la Petite Vallée $-\square$ écoles de la Grande Vallée - $\square$ écoles de périphérie

Cette configuration reflète les stratégies des écoles: derrière la situation moyenne des écoles Szent Endre $n^{\circ} 1$ et Márvány, se cache la bipolarité de ces écoles. Márvány organise une classe bilingue anglohongroise, et Szent Endre une classe " anglais-musique ", les deux classes de niveau sans doute les plus réputées parmi la classe moyenne de l'arrondissement au temps de notre recherche; mais les classes normales de ces écoles sont autant de classes "abandonnées ». Le cas de l'école Malom est très similaire : cet établissement présente également le cas de figure de "deux écoles en une seule ", mais le prestige général de cette école est plus modeste. Elle essaie d'attirer des enfants domiciliés hors de son secteur (de composition mixte, mais en moyenne modeste), avec cependant moins de succès que les deux autres écoles.

En contraste, Türr n'a nullement besoin de mettre en œuvre une logique d'action aussi agressive et active, puisqu'elle bénéficie dès le départ du meilleur secteur scolaire de l'arrondissement. Si elle ne cherche pas à attirer des enfants d'autres secteurs, c'est à la fois pour garder les «siens ", ne pas se brouiller avec les autres écoles... et accueillir les élèves issus des élites habitant l'arrondissement voisin, tout proche. D'ailleurs, l'autre école située tout près de cet établissement est celle de la Cité des Postiers, avec le milieu socioculturel le moins favorable de l'arrondissement et étiquetée comme «école tzigane ". Aussi, l'école Türr est à l'abri des migrations vers cet établissement voisin et, en même temps, peut compter sur les quelques familles plus ambitieuses du secteur voisin qui fuient l'école des Postiers. La proximité géographique et la distance socioculturelle des deux secteurs produisent donc une situation commode pour Türr, et aggravent la situation ségréguée de l'école des Postiers.

L'école Rutén montre l'exemple de la logique d'action d'une école active. Considérée comme la pire des écoles situées dans le centre, et ayant un secteur plutôt désavantagé, Rutén - menacée de fermeture vers 1995 - a décidé de réinterpréter ce prestige négatif en ouvrant une classe de rattrapage à effectif restreint. C'est ainsi que l'établissement, aux yeux 
Graphique 3. - Pourcentage d'élèves qui poursuivent leurs études dans les différents types de lycée

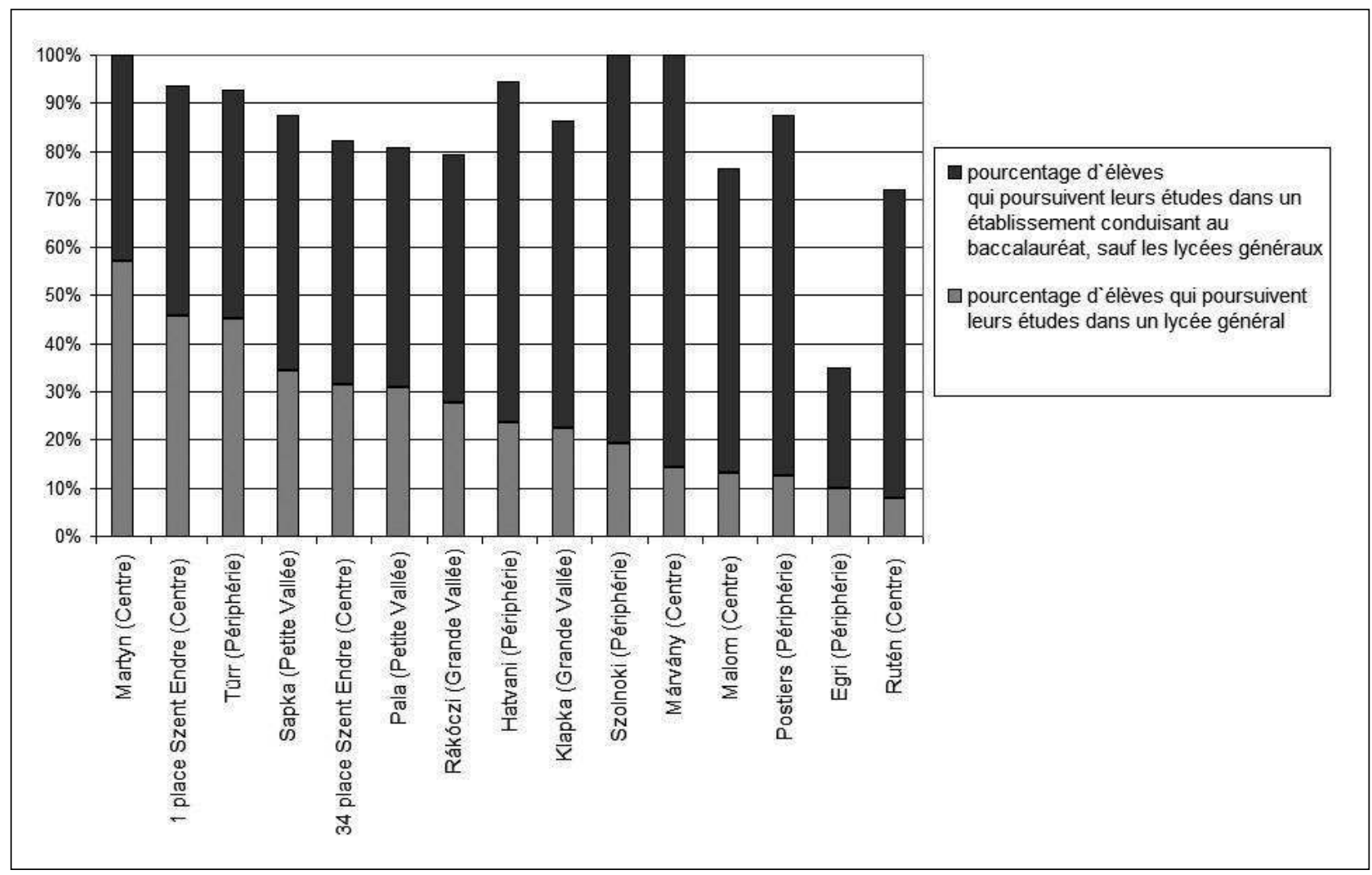

de la municipalité et des enseignants de l'arrondissement, est devenu un endroit où l'on s'occupe consciemment des enfants à problèmes, avec un personnel qualifié. L'école est fortement insérée dans le réseau d'interdépendance. Contrairement aux écoles favorisées, l'importance numérique des enfants résidant hors du secteur ne témoigne pas d'une attractivité et d'un choix délibéré de la part de ces familles; elle témoigne plutôt du fait que les différents acteurs (dont le centre de conseil pédagogique) profitent de la présence d'une école "spécialisée " où ils peuvent envoyer les enfants à problèmes sans rencontrer d'opposition. C'est ainsi que l'école, menacée autrefois de fermeture, a pu survivre en acceptant, comme l'indique le graphique 2, une composition sociale très défavorable. Autrement dit, il s'agit d'une école ségréguée, qui concentre un grand nombre d'exclus, Tsiganes et non-Tsiganes.

Quelle que soit la logique d'action d'une école donnée, du point de vue des élèves, un des enjeux majeurs est la poursuite des études dans le secondaire, et le type d'établissement qu'ils pourront fréquenter. Le calcul de la proportion d'élèves poursuivant des études dans un établissement d'enseignement secondaire (15) permet de classer les écoles selon les perspectives d'avenir qu'elles assurent à leurs élèves (voir graphique 3).

Le graphique illustre bien au niveau de l'arrondissement que même si l'accès aux écoles secondaires a tendance à se généraliser, cette évolution n'efface pas les inégalités de chance. Celles-ci apparaissent notamment lorsque l'on compare les proportions respectives d'élèves inscrits dans un lycée général à l'issue de l'école fondamentale - la voie royale vers le supérieur. À cet égard, les disparités demeurent énormes. Ajoutons à cela qu'il existe aussi des établissements dont beaucoup d'élèves (20 ou $30 \%$, voire $65 \%$ ) n'accèdent à aucune des filières secondaires conduisant au baccalauréat (lycée général ou lycée professionnel), ceux-ci poursuivent en général leurs études dans des écoles d'apprentissage sanctionnées d'un brevet professionnel. Nos données 
témoignent en outre de l'importance du secteur de recrutement. En effet, parmi les six écoles affichant de bons résultats du point de vue de la poursuite des études, aucune n'a un secteur de recrutement à composition sociale défavorable. Par ailleurs, la composition sociale de certaines écoles (du centre, par exemple) peut être encore plus favorable que celle de leur secteur de recrutement, en raison des élèves qu'elles attirent par leurs stratégies sélectives (examens d'admission, spécialisations «élitistes »). On voit aussi que ce que l'on appelle la ségrégation « bienveillante " des élèves à problèmes (qui consiste à les mettre dans les classes spéciales) coûte cher à l'école Rutén et aux enfants "ségrégués " qu'elle scolarise: ils ont beau entreprendre des études secondaires, ils ont très peu de chance d'être admis dans un lycée général.

La hiérarchie des écoles est cependant complexe et dépend de plusieurs facteurs. II s'agit en fait d'une stratification multidimensionnelle : il y a des différences entre les écoles (1) du point de vue de la composition sociale de leur environnement immédiat, ainsi que (2) du point de vue de la composition sociale réelle des effectifs d'élèves. Par ailleurs, indépendamment des données sociologiques, plusieurs autres facteurs entrent en jeu tels que (3) la position centrale ou périphérique d'un établissement à l'intérieur de l'espace local, (4) la taille de l'établissement (16), sans parler (5) des liens plus ou moins privilégiés qu'entretiennent les directeurs et directrices avec la mairie.

Un autre facteur s'ajoute à cette liste déjà longue ; il existe, aux yeux des parents, des écoles "à éviter à tout prix ". Ce rejet frappe avant tout les écoles scolarisant une proportion importante d'élèves tziganes. Ces établissements ne figurent jamais dans les projets scolaires des familles non tziganes qui ne choisissent pratiquement pas l'école des Postiers et, de plus en plus souvent, l'école Rutén. Le centre de conseil pédagogique de la mairie dépiste de plus en plus de «problèmes » - «dyslexie », « hyperactivité ", etc. ; les enfants tsiganes, majoritaires parmi les enfants dépistés, sont envoyés vers Rutén, et les non-Tsiganes vers l'autre établissement de Paladomb offrant une classe spéciale. C'est ainsi que la ségrégation des écoles peut aller en s'aggravant.

\section{CONCLUSIONS}

Les inégalités socio-géographiques à l'intérieur de l'espace urbain étudié, couplées à la liberté des parents de choisir eux-mêmes l'école fondamentale, engendrent nécessairement des inégalités interécoles. D'une part, certains établissements se spécialisent dans l'accueil d'un certain type d'élèves. Alors qu'autrefois les écoles essayaient surtout de se spécialiser dans l'accueil des enfants issus des couches sociales supérieures, on assiste aujourd'hui, du fait d'une forte concurrence et d'une nouvelle priorité politique allant de pair avec une incitation financière, à l'apparition d'écoles spécialisées dans le traitement des enfants en difficulté. On constate aussi que certains établissements agissent selon une logique de bipolarisation caractérisée par la coexistence au sein d'une même école de classes d'élites et de classes dites normales assurant un traitement ordinaire à des élèves, parmi lesquels nombreux sont les élèves en difficulté. Autrement dit, les logiques d'action adaptées au quasi-marché scolaire engendrent de la ségrégation à l'intérieur même des établissements, notamment en instaurant une "bipolarisation". Compte tenu de la composition sociale de l'arrondissement, globalement défavorable par rapport à la moyenne de la capitale, et du fait que les responsables de la politique éducative nationale et locale accordent des sommes supplémentaires lorsqu'il s'agit d'éduquer des enfants "à problèmes ", le marché scolaire est autant un " marché » d'élèves défavorisés qu'un " marché » d'élèves issus des strates sociales supérieures ou moyennes.

Certaines logiques d'action (par exemple, la création d'établissements ou de classes spécialisés dans l'accueil d'enfants “à problèmes "; le développement des prestations pédagogiques conçues en fonction d'un « handicap » dépisté chez l'enfant, etc. (17)) peuvent empêcher que les enfants en difficulté soient définitivement exclus du système scolaire ; elles peuvent même contribuer à améliorer leurs performances scolaires. En effet, le taux d'élèves défavorisés, y compris tsiganes, qui peuvent désormais passer leur baccalauréat augmente d'année en année. Toutefois, ces dispositifs et certaines pratiques pédagogiques, tout comme les programmes souvent inadaptés, peuvent avoir pour conséquence un renforcement de la ségrégation scolaire - non seulement au niveau du territoire étudié, mais aussi à l'intérieur même de certaines écoles - ainsi que des effets sociaux d'étiquetage des élèves «à problèmes " et des classes ou écoles qui les accueillent. Les phénomènes observés au niveau du territoire étudié doivent certainement être pris en compte lorsqu'il s'agit d'expliquer les résultats des récentes enquêtes PISA montrant un lien particulièrement fort en Hongrie entre origine sociale et performances des élèves. 
Ces inégalités très profondes résultent notamment des liens d'interdépendance très complexes que nous avons eu l'occasion de passer ici en revue. Dans cet arrondissement de la capitale hongroise (qui n'est atypique que parce qu'il n'y existe ni école privée, ni filière de lycée longue (18)), la concurrence entre établissements l'emporte le plus souvent sur la coopération : le tronc commun n'existe pratiquement pas (filières d'élite les plus diverses et classes de rattrapage coexistent dès le début de la scolarisation obligatoire) ; la mairie n'est jamais neutre et n'empêche pas les examens d'entrée par ailleurs illégaux ; la baisse démographique ne cesse de poser problème ; et enfin, de plus en plus d'enfants avec de potentielles " difficultés d'apprentissage " sont dépistés dès leur plus jeune âge par les institutrices de maternelle et le centre de conseil pédagogique de la mairie. Ces acteurs participent ainsi à la "co-régulation" des liens d'interdépendance, en complément de la régulation de marché basée sur le choix des parents.

Iván Bajomi

ibajomi@freemail.hu

Université Eötvös Loránd (ELTE), Budapest

Faculté de sciences sociales, Institut de sociologie

Eszter Berényi

beresz@webstation.hu

Ministère des affaires sociales et du travail, Budapest Mobilitás : centre pour la recherche de la jeunesse

Gábor Eröss

egabor@socio.mta.hu

Académie hongroise des sciences (MTA), Budapest Institut de sociologie (SzKi)

Anna Imre imrea@oki.hu

Institut national d'éducation publique (OKI), Budapest

\section{NOTES}

(1) Pour plus de détails concernant la décentralisation du système éducatif hongrois, voir Bajomi, 2002.

(2) Celle-ci se caractérise notamment par le libre choix des établissements, par l'existence de filières de haut niveau dès l'âge de l'obligation scolaire et aussi par la coexistence des écoles fondamentales accueillant des élèves de six à quatorze ans et des filières longues ouvertes depuis 1988 dans des lycées qui «écrèment " les meilleurs élèves des écoles fondamentales une fois que ceux-ci ont atteint l'âge de dix ou douze ans.

(3) Dans le cadre de cette enquête, nous avons d'une part réalisé un grand nombre d'entretiens semi-directifs. En dehors des responsables municipaux des questions scolaires, ont été interrogé les chefs d'établissements de toutes les écoles fondamentales de la municipalité et un grand nombre de professeurs et de parents d'élèves. En outre, des études de cas plus détaillées, complétées notamment d'observations, ont été réalisées dans deux écoles. Deux autres études de cas censées étudier de plus près le fonctionnement des instances locales chargées de gérer les établissements scolaires ont également été réalisées. D'autre part, nous avons collecté auprès des établissements des données concernant les inscriptions scolaires et nous avons aussi ré-exploité les résultats d'une action d'évaluation réalisée dans les écoles de l'arrondissement. De même, les données statistiques concernant la composition sociale des différents districts d'urbanisation ont également été prises en compte, tout comme un grand nombre de documents officiels et d'articles de presses ou comme plusieurs études concernant la scolarisation des enfants de l'arrondissement.

(4) Pour d'autres détails concernant les investigations menées en Hongrie dans le cadre du projet Reguledunetwork, voir Bajomi et al., 2004 \& 2006.

(5) Nous avons remplacé tous les noms désignant des institutions, des écoles ou l'arrondissement lui-même par des pseudonymes. Dans notre étude, nous analysons la situation de 2003 ; depuis, en raison notamment de la chute démographique, plusieurs écoles ont été fermées ou fusionnées.

(6) En 2004, une école de la cité (Klapka) a été fermée, son secteur partagé, ses élèves et ses enseignants dirigés vers Rákóczi.

(7) L'école privée «Arc-en-ciel » n'est pas non plus une école privée totalement indépendante de la municipalité. Comme il s'agit d'un établissement pour élèves " à problèmes ", la municipalité propose à quelques enfants de l'arrondissement de s'inscrire dans cette école. Pour ces enfants, ce n'est pas la famille, mais la municipalité, qui finance la scolarisation.

(8) Sur les questions de régulation, voir pour plus de détails Bajomi et al., 2004.

(9) Nous utilisons les données concernant les secteurs d'urbanisation. Celles-ci ne recoupent malheureusement qu'en partie les zones de recrutement.

(10) Les élèves venus d'autres arrondissement sont un facteur de déficit. En effet, le financement per capita versé par l'État ne couvre qu'à la hauteur de 60-70\% les dépenses de scolarisation, ainsi c'est l'arrondissement agissant en tant que propriétaire des écoles publiques et aussi en tant qu'autorité chargée d'assurer des services éducatifs qui doit compléter les subventions budgétaires. On comprend bien que la municipalité ne cofitions budgétaires. On comprend bien que la municipalité ne cofi-
nance pas volontiers la scolarisation d'élèves qui ne sont pas les enfants de leurs électeurs. Sauf lorsque des élèves venus d'autres arrondissements contribuent au maintien d'écoles ou de classes appelées à disparaître sans ce renfort. La subvention forfaitaire (ou per capita) est versée à la municipalité en fonction du nombre total d'élèves de l'ensemble des écoles de l'arrondissement. Ce chiffre est d'abord corrigé par le nombre d'élèves ayant des "besoins spécifiques " (dyslexie, difficultés d'apprentissage nécessitant l'inscription dans une classe à effectif réduit, etc.). Ensuite, la municipalité décide librement de suivre ou de ne pas suivre ces règles de financement officielles. Elle ne les suit jamais à la lettre et peut financer les classes bilingues de telle école aux dépens de classes «normales » de telle autre; de plus, les nombreux appels d'offre et autres appels à projets (du Ministère, de la capitale, des diverses Fondations, etc.) introduisent un autre facteur différentiel et inégalitaire.

(11) Les classes spécialisées élitistes étaient les suivantes en 2002 : classe bilingue anglais - hongrois (une école); musique vocale (deux écoles) ; sport (trois écoles); allemand (une école) ; anglais-informatique (deux écoles) ; art (une école ; en réalité : activités sportives et de danse) ; sport-études dites "récréation " (une école); classes dites «préparatoires » (trois écoles).

(12) Nous renvoyons pour une analyse plus détaillée de la question des inégalités à : Bajomi et al., 2006.

(13) Selon les données de l'enquête effectuée en 2001 par l'Institut pédagogique de Budapest, sur la base de questionnaires 
remplis par les élèves. Les réponses possibles étaient " 1 ": $8^{\mathrm{e}}$ classe de l'école fondamentale ; « 2 »: brevet d'apprentissage : "3»: baccalauréat (technique ou général). " 4 ": diplôme d'études supérieures. Le graphique représente l'ordre des écoles en fonction du niveau d'instruction moyen des pères. La moyenne de l'arrondissement est de 2,77 , c'est-à-dire inférieure au niveau du baccalauréat.

(14) Compte tenu de la baisse chronique des effectifs d'élèves, la municipalité a, dès le milieu des années 1990, voulu fermer un établissement scolaire à faibles effectifs, l'école Rutén. En vue d'échapper à cette menace, celle-ci a opté pour une spécialisation dans l'accueil des enfants en difficulté. Du seul point de vue de la survie de l'école, cette stratégie s'est avérée fort judicieuse. En effet, des nos jours, les pouvoirs locaux reçoivent une dotation per capita deux fois plus importante lorsqu'il s'agit de scolariser des enfants en difficulté. Ajoutons à cela qu'en 2003 l'arrondissement a procédé à une importante réorganisation de son réseau scolaire provoquant la fermeture et la fusion de plusieurs écoles. Signalons également qu'en cas de réorganisations scolaires, malgré leur statut de fonctionnaire, réorganisations scolaires, malgré leur statut de fonctionnaire,
les enseignants et les chefs d'établissement de Hongrie peuvent perdre leur emploi.

(15) En Hongrie, il existe trois formes majeures d'établissement secondaire: lycée général, lycée technique (tous les deux conduisent au baccalauréat) ou école professionnelle (qui ne délivre qu'un certificat d'aptitude professionnelle). Avec la proulaúat général, ce dernier tend à se dévaloriser relativement, tout comme le baccalauréat obtenu au terme d'études effectuées dans des lycées techniques. Le bac à lui seul ne garantit plus rien.

(16) Les écoles de très petite taille sont en effet menacées de fermeture, et certains parents le savent; l'incertitude concernant l'avenir est devenue en Hongrie un facteur d'inégalité à part entière pour les établissements scolaires.

(17) Sur les pratiques visant à dépister les handicaps chez les élèves et sur les phénomènes d'étiquetage liés à ceux-ci voir Berényi \& Eröss, 2003

(18) Ces filières longues s'emploient, dans la plupart des arrondissements de Budapest, à attirer les élèves issus des strates sociales supérieures ou moyennes à l'âge de dix ou douze ans, laissant " le reste " dans les écoles fondamentales jusqu'à l'âge de quatorze ans. Le cas de l'arrondissement étudié est donc dans ce sens un cas à part : compte tenu des distances à parcourir pour s'inscrire dans une "filière longue ", l'élite locale, dans sa grande majorité, préfère "coloniser " les écoles fondamentales du quartier jusqu'à la fin de l'école fondamentale.

\section{BIBLIOGRAPHIE}

BAJOMI I. (2002). «Décentralisation à la hongroise ». In I. Bajomi \& J.-L. Derouet (dir.), La grande récréation : la décentralisation de l'éducation dans six pays autrefois communistes. Paris: INRP, 2002, p. 41-71.

Bajomı I. ; BerénYI E. ; Eröss G. \& IMRE A. (2004). «Régulation scolaire dans un arrondissement ouvrier de Budapest ». Recherches sociologiques, $\mathrm{n}^{\circ} 2$, p. 103-121.

BAJOMI I. ; BerÉNYI E. ; ERÖSS G. \& IMRE A. (2006). « Compétition scolaire et inégalités dans un arrondissement de Budapest ". In C. Maroy, École, régulation et marché : une comparaison européenne. Paris : PUF [à paraître].

BAJOMI I. (2006). " L'école en Hongrie : entre transformations et instabilité ", Carrefours de l'éducation, n² 21, p. 126153.

BERÉNYI E. \& ERÖSS G. (2003). La naturalisation scolaire des inégalités sociales: ségrégation «bienveillante » dans un arrondissement ouvrier de Budapest. Conférence au $6^{\mathrm{e}}$ séminaire RAPPE : Paris, 13 décembre 2003

Cerych L. (1995). «Educational Reforms in Central and Eastern Europe », European Journal of Education, vol. $30, n^{\circ} 4$, p. 423-435.
ERöss G. (2006). «De l'illégal à l'inégal ou l'inverse ? Brève sociologie de l'école hongroise ", Nouvelle alternative : politique et société à l'Est, vol. 21, n 69-70 (à paraître).

HALÁsz G. (1994). "Les changements structurels dans le système d'enseignement hongrois ". Savoir, $\mathrm{n}^{\circ} 3$, p. 617-616.

HAVAS G. \& LISKó I (2005). « Szegregáció a roma tanulók általános iskolai oktatásában " [ "Ségrégation chez les élèves roms des écoles fondamentales »], Kutatás közben, $\mathrm{n}^{0} 266$

NÉMETH Sz. (2005). Chance for Integration. Integration vs. Segregation. Budapest: National Institute of Public Education, 2004.

VIRÁG T. (2003) “Gettósodó térség, gettósodó iskolarendszer » [ "Territoire et système scolaire en voie de ghettoïsation »]. In E. Kállai (dir.), A magyarországi cigány népesség helyzete a 21. század elején [La situation de la population tsigane de Hongrie à l'aube $d u X X I^{e}$ siècle]. Budapest: MTA Etnikai-nemzeti Kisebbségkutató Intézet, p. 127-138.

VAN ZANTEN A. (2001). L'école de la périphérie: scolarité et ségrégation en banlieue. Paris : PUF. 